\title{
Relationship between the Red Blood Cell Distribution Width and Risk of Acute Myocardial Infarction
}

\author{
Ping Wang, Yupeng Wang, Hongwei Li, Yongquan Wu and Hui Chen
}

Department of heart center Capital Medical University Affiliated Beijing Friendship Hospital, Beijing, China

\begin{abstract}
Aims: Recently, a number of studies have shown an increased red blood cell distribution width (RDW) to be a strong and independent predictor of the prognosis of coronary artery disease. The aim of this study was to investigate the underlying mechanisms responsible for the relationship between the RDW and a poor prognosis of coronary artery disease.

Methods: Four hundred and twenty-four patients with ST elevation myocardial infarction treated with primary percutaneous coronary intervention (pPCI) were analyzed retrospectively. We evaluated the relationships between the RDW and the high-sensitivity C-reactive protein (hsCRP) $\mathrm{N}$-terminal pro-brain natriuretic peptide (NTpro-BNP), fasting blood glucose and lipid levels, as well as other parameters of blood examinations and angiographic manifestations.

Results: There were 85 patients in the RDW $\geq 14 \%$ group (mean age $60.62 \pm 11.29$ years, and men: $87 \%$ ) and 339 patients in the RDW $<14 \%$ group (mean age: $59.74 \pm 11.55$ years, and men: $78 \%$ ). The RDW $\geq 14 \%$ group had higher platelet distribution width (PDW), NTpro-BNP and hsCRP values on admission, a heavier intracoronary thrombotic burden and a higher incidence of threebranch vascular lesions than the $\mathrm{RDW}<14 \%$ group. In the multiple logistic regression analysis, the associations between the RDW and the NTpro-BNP level, incidence of three-branch and left main lesions and intracoronary thrombotic burden remained.

Conclusions: A high RDW may be associated with the severity and instability of acute myocardial infarction.
\end{abstract}

JAtheroscler Thromb, 2015; 22:21-26.

Key words: Red blood cell distribution width, Acute myocardial infarction, Intracoronary thrombotic burden, Vascular lesion

\section{Introduction}

The red blood cell distribution width (RDW), measured using a blood analyzer, is a parameter of the volumetric variation of peripheral red blood cells (RBCs). Currently, it is primarily used to analyze the type of anemia and assist in the differential diagnosis of various leukemic conditions. In recent years, clinical trials have concluded that the RDW has prognostic value in patients with heart failure and coronary heart

Address for correspondence: Hui Chen, Department of heart center Capital Medical University Affiliated Beijing Friendship Hospital No.95 Yong'an Road, Xicheng District Beijing 100050, China

E-mail: wangpingyupeng@126.com

Received: January 20, 2014

Accepted for publication: June 25, 2014 disease $(\mathrm{CHD})^{1,2)}$. As demonstrated in previous studies, the RDW is correlated with CHD, peripheral atherosclerosis $^{3)}$ and diabetes ${ }^{4)}$, and the RDW values are markedly higher in acute myocardial infarction (AMI) patients than in those without a history of myocardial infarction $(\mathrm{MI})^{5}$. In addition, the RDW values are correlated with the in-hospital mortality of AMI patients undergoing direct $\mathrm{PCI}^{6}$, , AMI patients with a significantly elevated pre-discharge RDW compared to that observed on admission tend to have a higher mortality rate and worse prognosis ${ }^{7)}$. The above results indicate that the RDW is correlated with the occurrence and prognosis of AMI. However, the exact relationship between the RDW and AMI remains illdefined. Are changes in RDW actually secondary manifestations of AMI under a pathological state or does the RDW have predicative value for some patho- 
Table 1. Comparison of demographics and concurrent basal diseases

\begin{tabular}{|c|c|c|c|}
\hline & $\begin{array}{l}\text { RDW } \geq 14 \% \\
\quad \text { (in, } 85)\end{array}$ & $\begin{array}{c}\text { RDW }<14 \% \\
(\mathrm{n}, 339)\end{array}$ & $p$ value \\
\hline \multicolumn{4}{|l|}{ Demographic profiles } \\
\hline Age & $60.62 \pm 11.29$ & $59.74 \pm 11.55$ & 0.63 \\
\hline Gender (male/female) & $74 / 11$ & $264 / 75$ & 0.06 \\
\hline Smoking (yes/no) & $57 / 28$ & $216 / 123$ & 0.56 \\
\hline BMI $\left(\mathrm{kg} / \mathrm{m}^{2}\right)$ & $25.75 \pm 3.32$ & $25.13 \pm 3.99$ & \\
\hline $\log \mathrm{BMI}$ & $1.41 \pm 0.06$ & $1.40 \pm 0.06$ & 0.20 \\
\hline \multicolumn{4}{|l|}{ Basal diseases } \\
\hline Hypertension (yes/no) & $46 / 39$ & $196 / 143$ & 0.54 \\
\hline Diabetes (yes/no) & $17 / 68$ & $105 / 234$ & 0.13 \\
\hline Dyslipidemia (yes/no) & $17 / 68$ & $128 / 211$ & 0.002 \\
\hline Ischemic Cerebrovascular disease (yes/no) & $1 / 84$ & $17 / 322$ & 0.12 \\
\hline A previous history of angina (yes/no) & $25 / 60$ & $123 / 216$ & 0.24 \\
\hline SBP (mmHg) & $125.49 \pm 20.61$ & $122.94 \pm 22.01$ & \\
\hline $\log \mathrm{SBP}$ & $2.09 \pm 0.07$ & $2.08 \pm 0.09$ & 0.28 \\
\hline
\end{tabular}

SBP: systolic blood pressure

logical conditions associated with AMI? In this report, we observed and analyzed the clinical profiles of acute ST-segment elevation myocardial infarction (STEMI) patients undergoing primary percutaneous coronary intervention (pPCI) and examined the relationship between the laboratory test results and the RDW values so as to provide a new rationale for further elucidating the relationship between RDW and AMI.

\section{Methods}

\section{Study Subjects \\ Inclusion Criteria}

Hospitalized AMI patients undergoing pPCI at Beijing Friendship Hospital during the period of April 2009 to December 2012; men and women; no age limit; disease onset $<12$ hours; and suitability for emergency coronary angiography.

\section{Exclusion Criteria}

Previous hematological diseases, malnutrition, myocardial infarction, cardiomyopathy, rheumatic heart disease, chronic heart insufficiency, renal insufficiency, chronic pulmonary disease, hepatic disease, malignancy, immune disease, active inflammation, thyroid disease, an abnormal thyroid function, hormone use, currently dieting and a history of blood transfusion within the last three months.

\section{Study Parameters}

Age, gender, smoking status, body mass index (BMI) and concurrent basal diseases, including a his- tory of angina, hypertension, diabetes mellitus (DM), ischemic cerebrovascular disease and dyslipidemia. On admission, all values for blood pressure, white blood cell count (WBC), granulocytes (GR), lymphocytes (LY), RDW, hemoglobin (Hgb), platelet count (Plt), mean platelet volume (MPV), platelet distribution width (PDW) and N-terminal pro-brain natriuretic peptide (NTpro-BNP) were measured immediately. In addition, the levels of thyroid-stimulating hormone (TSH), high-sensitivity C-reactive protein (hsCRP), fasting blood glucose (GLU), high-density lipoprotein-cholesterol (HDL-C), low-density lipoproteincholesterol (LDL-C), total glycerides (TG) and total cholesterol (TC) were obtained from fasting blood specimens the morning after admission. Blood was routinely measured using a Sysmex XE2100 hematology analyzer.

\section{Intracoronary Thrombotic Burden Analysis}

Intracoronary thrombi were angiographically identified as previously described: thrombus grade 0 (G0), no angiographic characteristics of thrombi were present; thrombus grade 1 (G1), possible thrombi were present with angiographic characteristics such as a reduced contrast density, haziness, irregular lesion contour or a smooth convex meniscus at the site of total occlusion suggestive but not diagnostic of a thrombus; definite thrombi (G2-4); total vessel occlusion (G5).

\section{Severity of Coronary Lesions}

The severity of coronary lesions was assessed 
Table 2. Comparison of laboratory data between the RDW $\geq 14 \%$ and RDW $<14 \%$ groups

\begin{tabular}{lccc}
\hline & RDW $\geq 14 \%(\mathrm{x} \pm \mathrm{s})$ & RDW $<14 \%(\mathrm{x} \pm s)$ & $p$ value \\
\hline WBC $\left(\times 10^{9} / \mathrm{L}\right)$ & $10.44 \pm 3.29$ & $11.38 \pm 3.52$ & 0.26 \\
GR $\left(\times 10^{9} / \mathrm{L}\right)$ & $8.22 \pm 3.01$ & $9.0 \pm 43.32$ & 0.37 \\
RBC $\left(\times 10^{12} / \mathrm{L}\right)$ & $4.55 \pm 0.74$ & $4.56 \pm 0.56$ & 0.88 \\
PLT $\left(\times 10^{9} / \mathrm{L}\right)$ & $229.0 \pm 763.15$ & $223.83 \pm 57.86$ & 0.46 \\
MPV $(\mathrm{fl})$ & $8.91 \pm 0.98$ & $9.74 \pm 1.14$ & $<0.001$ \\
PDW $(\%)$ & $14.26 \pm 2.60$ & $12.90 \pm 12.40$ & $<0.001$ \\
$\operatorname{logPDW}$ & $1.14 \pm 0.08$ & $1.10 \pm 0.08$ & 0.02 \\
TC $(\mathrm{mmol} / \mathrm{L})$ & $4.60 \pm 0.94$ & $4.87 \pm 1.03$ & 0.33 \\
HDL $(\mathrm{mmol} / \mathrm{L})$ & $0.91 \pm 0.21$ & $0.94 \pm 0.25$ & 0.006 \\
LDL $(\mathrm{mmol} / \mathrm{L})$ & $2.55 \pm 0.67$ & $2.80 \pm 0.68$ & $1.14 \pm 1.41$ \\
TSH (uIU/mL) & $1.13 \pm 1.14$ & $-0.14 \pm 1.41$ & 0.68 \\
$\log$ TSH & $-0.11 \pm 0.41$ & $316.62 \pm 1155.42$ & \\
NTpro-BNP (ng/dL) & $632.80 \pm 1380.34$ & $1.78 \pm 0.73$ & \\
Log NTpro-BNP (ng.dL) & $2.20 \pm 0.74$ & $3.49 \pm 0.41$ & 0.001 \\
LA (cm) & $3.58 \pm 0.04$ & $0.54 \pm 0.05$ & 0.07 \\
$\operatorname{logLA}(\mathrm{cm})$ & $0.55 \pm 0.04$ & $5.34 \pm 0.47$ & 0.96 \\
EDD $(\mathrm{cm})$ & $5.34 \pm 0.52$ & & \\
\hline
\end{tabular}

based on $>50 \%$ stenosis in the coronary arteries.

\section{Statistical Analysis}

The SPSS11.5 statistical software program was employed for the statistical analysis. Quantitative data were assessed using the $\chi^{2}$ test, and quantitative data in a normal distribution were expressed as $x \pm s$. Intergroup differences were analyzed with the $t$-test, and quantitative data in a non-normal distribution were expressed as the rank-mean. Intergroup differences were analyzed with the rank-sum test.

\section{Results}

A total of 424 patients were recruited, including 85 patients in the RDW $\geq 14 \%$ group and 339 patients in the RDW $<14 \%$ group. The demographics and baseline diseases were compared between the two groups (Table 1). Consequently, there were no differences in age, gender, BMI, SBP or a previous history of smoking, hypertension, diabetes, dyslipidemia, angina or ischemic cerebrovascular disease between the RDW $\geq 14 \%$ group and the RDW $<14 \%$ group. However, the number of patients with a history of dyslipidemia was markedly smaller in the RDW $\geq 14 \%$ group than in the RDW $<14 \%$ group.

Parametric comparison of routine blood parameters, including TC, TSH, NTpro-BNP, left atrial (LA) and end-diastolic diameter (EDD) between the RDW $\geq 14 \%$ and RDW $<14 \%$ groups. The platelet distribution width (PDW) and NTpro-BNP values were markedly higher in the RDW $\geq 14 \%$ group than in the $\mathrm{RDW}<14 \%$ group, whereas the mean platelet volume (MPV), TC and low-density lipoprotein (LDL) values were lower in the RDW $\geq 14 \%$ group than in the RDW $<14 \%$ group (Table 2).

Comparison of the severity of coronary lesions and thrombotic burden between the RDW $\geq 14 \%$ and RDW $<14 \%$ groups. The RDW $\geq 14 \%$ group had a heavier intracoronary thrombotic burden and higher percentage of three-branch and left main lesions (Table 3).

Comparison of parameters with a non-normal distribution between the RDW $\geq 14 \%$ and RDW $<14 \%$ groups. Compared with the RDW $<14 \%$ group, the RDW $\geq 14 \%$ group had higher levels of hsCRP (Table 4).

Comparison of cardiac events during hospitalization between the RDW $\geq 14 \%$ and RDW $<14 \%$ groups. There were no significant differences (Table 5).

According to the logistic regression analysis, the NTpro-BNP level and angiographic presence of thrombi and/or vascular lesions (three-branch or left main) were independently correlated with the RDW (Table 6).

\section{Discussion}

In the present study, we examined the association between the RDW values and other clinical profiles of acute ST-segment elevation myocardial infarction (STEMI) patients undergoing primary percutaneous 
Table 3. Comparison of vascular lesions between the two groups

\begin{tabular}{lccc}
\hline & RDW $\geq 14 \%$ & RDW $<14 \%$ & $p$ value \\
\hline Thrombotic burden (G0/G1-5) & $8 / 77$ & $67 / 272$ & 0.03 \\
Vascular lesion (1/2/3-branch or left main) & $19 / 25 / 41$ & $147 / 110 / 82$ & $<0.001$ \\
Vascular lesion (1/2-branch) & $19 / 25$ & $147 / 110$ & 0.08 \\
Vascular lesion (1/3-branch or left main) & $19 / 41$ & $147 / 82$ & $<0.001$ \\
Vascular lesion (2/3-branch or left main) & $25 / 41$ & $110 / 82$ & 0.01 \\
\hline
\end{tabular}

Table 4. Comparison of various parameters with a non-normal distribution

\begin{tabular}{lccccccc}
\hline & Onset time & DBP & hsCRP & LY & Hgb & TG & GLU \\
\hline Mann-Whitney U & 13921 & 13333.5 & 11083 & 14362 & 12881 & 13775.5 & 13308 \\
Wilcoxon W & 17576 & 16903.5 & 68713 & 71992 & 16536 & 17345.5 & 16878 \\
Z & -.485 & -0.905 & -3.145 & -.045 & -0.421 & -1.511 & -.808 \\
$p$ value & .63 & .37 & .002 & 0.96 & 0.47 & 0.13 & .42 \\
\hline
\end{tabular}

DBP: diastolic blood pressure, hsCRP: high-sensitivity C-reactive protein, LY: lymphocytes, Hbg: hemoglobin, TG: triglycerides, GLU: fasting blood glucose

coronary intervention (pPCI) and found that the RDW $\geq 14 \%$ group had a heavier burden of intracoronary thrombi. Additionally, the patients with higher RDW values had more severe coronary lesions, consistent with the findings of Akin F. et al. ${ }^{8}$. The patients with an RDW of $\geq 14 \%$ also higher levels of PDW, indicating an increased platelet activity and prethrombotic state, as well as higher levels of NTproBNP, a marker of the cardiac functional status and cardiac load. A relationship between RDW and BNP was recently reported in patients with coronary artery disease (CAD) by Fukuta H. et al. ${ }^{9}$, and we showed in the present study that an elevated RDW is independently associated with a high NTpro-BNP level in patients with acute STEMI.

The RDW is a parameter of variation in the $\mathrm{RBC}$ volume. An increased RDW value is primarily correlated with the acceleration of RBC proliferation, as the release of larger reticulocytes into the circulation increases the RDW and any changes influencing the production of RBC may bring about alterations in the RDW. An increased RDW is likely correlated with the inflammatory response, and various inflammatory factors, such as granulocyte colony-stimulating factor and erythropoietin, may stimulate the proliferation of $\mathrm{RBC}$, thus increasing the $\mathrm{RDW}^{10)}$. Inflammatory responses may also interfere with iron metabolism, thereby reducing the lifespan and lowering the plasticity of circulating RBCs, as well as modifying the responses of bone marrow to erythropoietin and increasing the size of $\mathrm{RBCs}^{11)}$, ultimately increasing the RDW. Lippi G. et al. evaluated a total of 3,845
Table 5. Comparison of cardiac events during hospitalization

\begin{tabular}{lccc}
\hline & RDW $\geq 14 \%$ & RDW $<14 \%$ & $p$ value \\
\hline Heart failure event & $6 / 79$ & $16 / 323$ & 0.39 \\
Re-ischemia & $1 / 84$ & $6 / 333$ & 0.70 \\
Arrhythmia & $12 / 73$ & $35 / 304$ & 0.32 \\
Cardiogenic mortality & $1 / 84$ & $7 / 332$ & 0.59 \\
\hline
\end{tabular}

hospitalized patients in order to explore the correlation between the RDW and the inflammatory factors, the hsCRP level and the erythrocyte sedimentation rate (ESR). Consequently, a multivariable regression analysis revealed a graded correlation between the RDW values and both the hsCRP and ESR values, independent of age, gender, the mean RBC volume, hemoglobin and ferritin ${ }^{12)}$. Meanwhile, Akin F. et al. assessed AMI patients and found the RDW to be positively correlated with the neutrophil/lymphocyte ratio $\left.(r=0.178, p<.001)^{8}\right)$. As demonstrated in the study of Vaya A. et al., the RDW has also been found to be positively correlated with the neutrophil count in AMI patients ${ }^{13)}$. In the present study, the STEMI patients with an RDW of $\geq 14 \%$ had higher levels of hsCRP, indicating that an altered RDW value is correlated with the inflammatory response. The activation of the neuroendocrine system has an effect on the production of $\mathrm{RBCs}^{14)}$ and thus the RDW. As a marker of neurohumoral activation, the NTpro-BNP also reflects the cardiac load. In the present study, the levels of NTpro-BNP were markedly higher in the RDW $\geq 14 \%$ group than in the RDW $<14 \%$ groups, which 
Table 6. Logistic regression analysis of the relationship between the RDW values and the hsCRP and NTpro-BNP levels and angiographic presence of thrombi

\begin{tabular}{lccccc}
\hline & B & SE & Wald & $p$ value & OR \\
\hline NTpro-BNP & 0.649 & 0.270 & 5.769 & 0.016 & 1.914 \\
hsCRP & 0.404 & 0.259 & 2.422 & 0.120 & 1.497 \\
angiographic presence of thrombus & 0.811 & 0.390 & 4.331 & 0.037 & 2.250 \\
Vascular lesion (3-branch or left main) & 0.952 & 0.245 & 15.150 & 0.000 & 2.592 \\
\hline
\end{tabular}

RDW: red blood cell distribution width, hsCRP: high-sensitivity C-reactive protein, NTpro-BNP: N-terminal pro-brain natriuretic peptide

suggests that the RDW is a marker of the cardiac load and neurohumoral activation in AMI patients.

Furthermore, the RDW may be correlated with the formation and stability of atherosclerotic plaque ${ }^{15)}$. Akin F. et al. used the Syntax score to evaluate the degree of coronary atherosclerosis and showed, in a logistic regression analysis, that the RDW is correlated with a high Syntax score in AMI patients (odds ratio (OR): 1.165$)^{9)}$. In the present study, the STEMI patients with an RDW of $\geq 14 \%$ had a higher percentage of three-branch and left main lesions. These results are consistent with those of Akin F. et al. The cholesterol-rich RBC membrane is likely to seep into sites of atherosclerotic plaque, and membranous cholesterol in RBCs is a major source of the atheromatous core ${ }^{16)}$. Recently, the total content of RBC membranous cholesterol was found to be correlated with coronary instability. Therefore, membranous cholesterol in RBCs has been proposed to be a new marker of acute coronary syndrome ${ }^{17)}$. Furthermore, the content of membranous cholesterol in RBCs determines the plasticity of the RBC membrane and the morphology and dimensions of the cell. A recent study by Tziakas D. et al. showed that the RDW is independently positively correlated with the level of RBC membranous cholesterol $^{18)}$. Therefore, the RDW is likely correlated with plaque instability. The major manifestations of plaque instability include plaque tearing and thrombosis. The initial tear in an atherosclerotic plaque manifests as the intraplaque hemorrhage of $\mathrm{RBCs}^{19)}$. RBCs and serum iron also participate in the process of oxidative stress. The lifespan of a RBC is shortened by oxidative stress, such that immature RBCs are released into the bloodstream. As a result, oxidative stress leads to poor membrane plasticity of RBCs. The size of RBCs varies, and an increased RDW is correlated with reduced antioxidant effects ${ }^{20)}$. Therefore, an increased RDW may indicate a state of oxidative stress. Moreover, oxidative stress is correlated with both the formation and tearing of atherosclerotic plaques in the coronary arteries, and an elevated RDW is correlated with the no-reflow phenomenon on post-PCI ECG in AMI patients ${ }^{21)}$. Karabulut A. et al. analyzed the relationship between the RDW and the post-PCI TIMI flow in AMI patients and found an RDW of $>14.8 \%$ on admission to be independently correlated with poor coronary perfusion ${ }^{22)}$. The thrombotic burden is a major mechanism underlying poor coronary perfusion after PCI. Early-onset AMI patients have higher RDW values than their same-age counterparts without AMI, and early-onset MI is correlated with coronary spasms and thrombosis ${ }^{23)}$. In the present study, the patients with higher RDW values tended to have higher PDW levels. A high PDW level reflects a large degree of variation in the size of platelets and a high platelet activity, as well as being a marker of a prothrombotic state ${ }^{24)}$. The above study results hint that the RDW may be correlated with the formation of intracoronary thrombi and demonstrated a more severe intracoronary thrombotic burden in the STEMI patients with an RDW of $\geq 14 \%$. Therefore, the RDW may be a marker of plaque instability and the formation/consistency of intracoronary thrombi. In STEMI patients with severe and complicated coronary lesions, a greater thrombotic burden results in greater activation of the neuroendocrine system and subsequently a heavier cardiac load. Therefore, the more severe coronary lesions and greater thrombotic burden observed in the patients with an RDW of $\geq 14 \%$ in the present study are consistent with the effects of a higher level of NTpro-BNP.

The measurement of RDW has been incorporated into routine blood examinations. As a simple, readily available and cost-effective laboratory parameter, the RDW is likely to become a rapid and intuitive tool for risk stratification of STEMI patients. In addition, a high RDW value may reflect the severity and instability of STEMI.

\section{Limitations}

This single-center, retrospective trial failed to consider the effects of serum iron and vitamin 12 on the RDW values. 


\section{Funding}

This work was supported by the capital health research and development projects, Beijing, China.

\section{Conflicts of Interest}

There are no conflicts of interest to declare in association with this study.

\section{References}

1) Felker GM, Allen LA: Red cell distribution width as a novel prognostic marker in heart failure: data from the CHARM Program and the Duke databank for cardiovascular diseases. Am Coll Cardiol, 2007; 50: 402-407

2) Tonelli M, Sacks F, Arnold M, Moye L, Davis B, Pfeffer $\mathrm{M}$; for the Cholesterol and Recurrent Events (CARE) Trial Investigators: Relation between red blood cell distribution width and cardiovascular event rate in people with coronary disease. Circulation, 2008; 117: 163-168

3) Wen Y: High red blood cell distribution width is closely associated with risk of carotid artery atherosclerosis in patients with hypertension. Exp Clin Cardiol, 2010; 15: $37-40$

4) Malandrino N, Wu WC, Taveira TH, Whitlatch HB, Smith RJ: Association between red blood cell distribution width and macrovascular and microvascular complications in diabetes. Diabetologia, 2012; 55: 226-235

5) Cemin R, Donazzan L, Lippi G, Clari F, Daves M: Blood cells characteristics as determinants of acute myocardial infarction. Clin Chem Lab Med, 2011; 49: 1231-1236

6) İlhan E, Güvenç TS, Altay $S$, Çağdaş $M$, Çalik AN, Karaca M, Güzelburç Ö, Karaca G, Biteker M, Tayyareci G: Predictive value of red cell distribution width in intrahospital mortality and postintervention thrombolysis in myocardial infarction flow in patients with acute anterior myocardial infarction. Coron Artery Dis, 2012; 23: 450454

7) Dabbah S, Hammerman H, Markiewicz W, Aronson D: Relation between red cell distribution width and clinical outcomes after acute myocardial infarction. Am J Cardiol, 2010; 105: 312-317

8) Akin F, Köse N, Ayça B, Katkat F, Duran M, Uysal OK, Arinc H: Relation between red cell distribution width and severity of coronary artery disease in patients with acute myocardial infarction. Angiology, 2013; 64: 592-596

9) Fukuta H, Ohte N, Mukai S, Saeki T, Asada K, Wakami K, Kimura G: Elevated plasma levels of B-type natriuretic Peptide but not C-reactive protein are associated with higher red cell distribution width in patients with coronary artery disease. Int Heart J, 2009; 50: 301-312

10) Caramelo C, Justo S, Gil P: Anemia in heart failure: pathophysiology, pathogenesis, treatment, and incognitae. Rev Esp Cardiol, 2007; 60: 848-860

11) Weiss G, Goodnough LT: Anemia of chronic disease. N Engl J Med, 2005; 352: 1011-1023
12) Lippi G, Targher G, Montagnana M, Salvagno GL, Zoppini G, Guidi GC: Relation between red blood cell distribution width and inflammatory biomarkers in a large cohort of unselected outpatients. Arch Pathol Lab Med, 2009; 133: 628-632

13) Vaya A, Hernández JL, Zorio E, Bautista D: Association between red blood cell distribution width and the risk of future cardiovascular events. Clin Hemorheol Microcirc. 2012; 50: 221-225

14) Cole J, Ertoy D, Lin H, Sutliff RL, Ezan E, Guyene TT, Capecchi M, Corvol P, Bernstein KE: Lack of angiotensin II-facilitated erythropoiesis causes anemia in angiotensinconverting enzyme deficient mice. J Clin Invest, 2000; 106: $1391-1398$

15) Tziakas DN, Chalikias GK, Stakos D, Boudoulas H: The role of red blood cells in the progression and instability of atherosclerotic plaque. Int J Cardiol, 2010; 142: 2-7

16) Pasterkamp G, Virmani R: The erythrocyte: new player in atheromatous core formation. Heart, 2002; 88: 115-116

17) Zhong Y, Tang H, Zeng Q, Wang X, Yi G, Meng K, Mao Y, Mao X: Total cholesterol content of erythrocyte membranes is associated with the severity of coronary artery disease and the therapeutic effect of rosuvastatin. Ups J Med Sci, 2012; 117: 390-398

18) Tziakas D, Chalikias G, Grapsa A, Gioka T, Tentes I, Konstantinides S: Red blood cell distribution width: a strong prognostic marker in cardiovascular disease: is associated with cholesterol content of erythrocyte membrane. Clin Hemorheol Microcirc, 2012; 51: 243-254

19) Kolodgie FD, Gold HK, Burke AP, Fowler DR, Kruth HS, Weber DK, Farb A, Guerrero LJ, Hayase M, Kutys R, Narula J, Finn AV, Virmani R: Intraplaque hemorrhage and progression of coronary atheroma. N Engl J Med, 2003; 349: 2316-2325

20) Semba RD, Patel KV, Ferrucci L, Sun K, Roy CN, Guralnik JM, Fried LP: Serum antioxidants and inflammation predict red cell distribution width in older women: Women's Health and Aging Study I. Clin Nutr, 2010; 29: 600604

21) Isik T, Kurt M, Ayhan E, Tanboga IH, Ergelen M, Uyarel $\mathrm{H}$ : The impact of admission red cell distribution width on the development of poor myocardial perfusion after primary percutaneous intervention. Atherosclerosis, 2012; 224: 143-149

22) Karabulut A, Uyarel H, Uzunlar B, Çakmak M: Elevated red cell distribution width level predicts worse postinterventional thrombolysis in myocardial infarction flow reflecting abnormal reperfusion in acute myocardial infarction treated with a primary coronary intervention. Coron Artery Dis, 2012; 23: 68-72

23) Uysal OK, Duran M, Ozkan B, Sahin DY, Tekin K, Elbasan Z, Akin F, Balli M, Gunebakmaz O, Arinc H, Kaya MG, Cayli M: Red cell distribution width is associated with acute myocardial infarction in young patients. Cardiol J, 2012; 19: 597-602

24) Vagdatli E, Gounari E, Lazaridou E, Katsibourlia E, Tsikopoulou F, Labrianou I: Platelet distribution width: a simple, practical and specific marker of activation of coagulation. Hippokratia, 2010; 14: 28-32 